\title{
Entreciencias
}

Entreciencias: diálogos en la Sociedad del Conocimiento

ISSN: 2007-8064

entreciencias@enes.unam.mx

Universidad Nacional Autónoma de México México

\section{Innovación docente en la Educación Superior Intercultural: notas introductorias al número especial}

Dietz, Gunther; Mateos Cortés, Laura Selene

Innovación docente en la Educación Superior Intercultural: notas introductorias al número especial

Entreciencias: diálogos en la Sociedad del Conocimiento, vol. 5, supl. 14, 2017

Universidad Nacional Autónoma de México, México

Disponible en: http://www.redalyc.org/articulo.oa?id=457652442001

DOI: http://dx.doi.org/10.21933/J.EDSC.2017.E1

Se autoriza la reproducción total o parcial de los textos aquí publicados siempre y cuando se cite la fuente completa y la dirección electrónica de la publicación

Esta obra está bajo una Licencia Creative Commons Atribución-NoComercial-SinDerivar 4.0 Internacional. 


\section{Innovación docente en la Educación Superior Intercultural: notas introductorias al número especial}

Teaching innovation in Intercultural High Education: introductory notes to the Special Issue

Gunther Dietz guntherdietz@gmail.com Universidad Veracruzana, Alemania

Laura Selene Mateos Cortés lauramat@gmail.com

Universidad Veracruzana, México

Entreciencias: diálogos en la Sociedad del Conocimiento, vol. 5, supl. 14, 2017

Universidad Nacional Autónoma de México, México

DOI: http://dx.doi.org/10.21933/ J.EDSC.2017.E1

Redalyc: http://www.redalyc.org/ articulo.oa?id=457652442001

Se autoriza la reproducción total o parcial de los textos aquí publicados siempre y cuando se cite la fuente completa y la dirección electrónica de la publicación. CC BY-NC-ND

\section{Innovación docente en la Educación Superior Intercultural: notas introductorias al número especial}

La innovación educativa en general y la innovación docente en particular, se han convertido en una panacea aclamada por todo tipo de actores educativos, gubernamental y no -gubernamental, público y privado, que interviene en los debates contemporáneos sobre la educación y las reformas educativas. Tanto en la educación escolar básica, como en la educación superior, la innovación se vuelve prácticamente un sinónimo de "modernización” y de "tecnologización" educativa. Frente a este uso reduccionista- y obviamente influido por intereses relacionados con la mercantilización de determinados procesos áulicos-, reivindicamos una comprensión más crítica y holística de la innovación como proceso de cambio educativo, que se inspira en la pedagogía alternativa, comprometida con la transformación integral de las instituciones escolares. En este sentido, recuperamos la definición de innovación educativa, que propone en sus "Pedagogías del siglo XXI" Carbonell Sebarroja, entendiéndolas como todas aquellas que

\footnotetext{
... se ocupan y preocupan por mejorar las relaciones educativas y lograr un entorno escolar más amable; por fomentar una mayor cooperación, participación y democratización de la gestión diaria de la escuela; por estimular el protagonismo del alumnado y su curiosidad por el conocimiento; por convertir el aula en un espacio de investigación y conversación; por acercar la escuela a la realidad y ésta a la escuela para conseguir que cuanto se enseña y aprende en la escuela sea estimulante y tenga un sentido para la formación de una futura ciudadanía más libre, responsable, creativa, crítica y responsable; con el fin de que se active al propio tiempo el desarrollo del pensamiento y del conjunto de sentimientos; y, en definitiva, para que la escuela sea más educativa. (Carbonell, 2015, p.15)
}

Específicamente en el ámbito de la educación superior, desde los años noventa, la institución universitaria se ve expuesta a nivel internacional a crecientes exigencias de innovación. En el marco de discursos de "modernización universitaria" y de "internacionalización", se ejerce presión gubernamental y empresarial de orientación neoliberal, para que las instituciones de educación superior (IES) se adapten a 
criterios de empleabilidad de sus egresadas y egresados, de auditorías internas y externas y de evaluaciones periódicas de sus "estándares de calidad" (Ibarra, 2002; Shore y Wright, 2015).

En este contexto, la innovación educativa en las universidades, se suele reflejar en demandas por explicitar las didácticas implícitas y consuetudinarias que rigen la enseñanza universitaria y, por modificar sus tradicionales estilos monológicos de "impartir cátedra" mediante metodologías de trabajo colaborativo, centradas en los procesos de aprendizaje de las y los estudiantes, con un incremento de actividades prácticas a lo largo de toda la carrera universitaria y una mayor vinculación entre momentos áulicos y experiencias extra-áulicas (Clark, 2000; Inayatullah y Gidley, 2003; Gazzola y Didriksson, 2008). Para explorar el potencial transformador de este tipo de innovación en cuanto al surgimiento de tipos alternativos de IES, de "universidades del siglo XXI" (Boaventura de Sousa, 2005), en el presente número especial de la revista Entreciencias. Diálogos en la Sociedad del Conocimiento, pretendemos describir e ilustrar casos concretos de innovación docente introducidos en las llamadas "Instituciones Interculturales de Educación Superior" (Mato, 2009), nombradas así en la última década, y representadas en México, sobre todo, por las así denominadas universidades interculturales.

A más de diez años de su creación, estas universidades interculturales mexicanas atienden a jóvenes estudiantes de origen rural y/o indígena en sus propias regiones mediante carreras universitarias pretendidamente pensadas, diseñadas e implementadas desde las realidades educativas, culturales y lingüísticas de los pueblos originarios. En los últimos años, hemos visto cómo en el Estado de México, Chiapas, Veracruz, Tabasco, Puebla, Quintana Roo, Michoacán, Guerrero, San Luis Potosí, Hidalgo y Sinaloa, surgen estas nuevas ies, en su gran mayoría, al amparo de la Coordinación General de Educación Intercultural y Bilingüe (CGEIB) de la SEP, a través de convenios realizados entre la federación y los gobiernos estatales (Casillas y Santini, 2006). A estas universidades interculturales, se suman en algunos estados -Oaxaca, Chiapas, Michoacán, Sinaloanuevas escuelas normales de enfoque intercultural bilingüe, orientadas a la formación de docentes de educación básica, hablantes de una lengua indígena, para impartir clases a nivel preescolar y/o primaria bilingüe e intercultural en sus regiones y comunidades de origen (Mateos, Dietz y Mendoza, 2013).

Sobre este aún incipiente subsistema de educación superior intercultural, como se comienza a denominar, se ha escrito y publicado mucho en los últimos años, ${ }^{1}$ particularmente, sobre los procesos de creación e institucionalización de las universidades interculturales, sobre los conflictos que desde su origen albergan: entre intereses académicos vs. intereses político-partidistas, entre actores comunitarios vs. externos, entre tendencias hacia la burocratización vs. tendencias hacia la academización y/o la vinculación comunitaria de las universidades interculturales. En toda una serie de investigaciones externas, autoinvestigaciones, sistematizaciones y productos de tesis de posgrado que 
hemos procurado sistematizar y poner en diálogo a través de estados de conocimiento (cfr. Mateos y Dietz, 2013, 2016) y por medio de seminarios de investigación anualmente celebrados a nivel nacional (cfr. Cuerpo Académico Estudios Interculturales (2016a, 2016b), percibimos que hay un aspecto apenas estudiado, escasamente visibilizado: la práctica docente que se lleva a cabo al interior de las universidades interculturales, su especificidad en comparación con los procesos más convencionales y áulicos de enseñanza y aprendizaje universitaria y su articulación de actores estudiantiles, docentes y comunitarios.

Es por ello, que para este número especial, hemos convocado a académicas/os que trabajen con universidades interculturales y escuelas normales bilingües interculturales para que aporten artículos de investigación y/o sistematización sobre la innovación docente en la educación superior intercultural. El rico abanico de estudios de caso que a continuación aportan las y los autores, reflejan fehacientemente los diferentes modelos de ies interculturales que a la fecha existen en el país. En la primera parte, contamos con investigaciones enfocadas hacia tres tipos de universidades interculturales: universidades creadas por convenio con la CGEIB, universidades de creación comunitaria y universidades interculturales creadas por universidades autónomas pre-existentes. Mientras que el artículo de Carlos Montalvo Martínez proviene de una universidad intercultural prototípica, la Universidad Intercultural Indígena de Michoacán (UIIM), creada por un convenio entre el Gobierno del Estado de Michoacán y la CGEIB de la sep federal, el trabajo de Carlos Luis Maldonado Ramírez analiza una experiencia de la Unidad de Estudios Superiores de Alotepec, Mixe (UESA), en Oaxaca, una ies comunitaria creada al margen de la CGEIB y cuya carrera se encuentra en proceso de homologación con el subsistema intercultural; por último, contamos con cuatro estudios de caso -aportados por Cuauhtémoc Jiménez Moyo, Sara Itzel Arcos Barreiro, Claudia Eguiarte Espejo y Carlos Alberto Cruz González- provenientes de la Universidad Veracruzana Intercultural (UVI), una entidad intercultural reconocida por la CGEIB, pero que forma parte de la Universidad Veracruzana, y que como tal, goza de autonomía frente a su respectivo gobierno del estado. En la segunda parte, estos trabajos son complementados por dos estudios de caso provenientes de ies formadoras de docentes en educación básica: Benjamín Maldonado Alvarado presenta innovación docente que se está desarrollando en la Escuela Normal Bilingüe e Intercultural de Oaxaca (ENBIO), mientras que María Isabel Ramírez Duque analiza el lugar de los saberes indígenas en una especialidad de educación intercultural bilingüe ofrecida por la Universidad Pedagógica Experimental Libertador de Venezuela.

En concreto, el artículo que abre este número especial, "Formarse para transformar: valoración del proceso formativo de estudiantes de la Universidad Veracruzana Intercultural (UVI)", de Cuauhtémoc Jiménez Moyo, constituye una especie de meta-investigación crítica que reconstruye y evalúa cómo las y los estudiantes de la Sede Regional Grandes Montañas de la UVI llevan a cabo sus propios proyectos de 
investigación vinculada con actores comunitarios que desembocan en sus documentos recepcionales. Con este énfasis, el autor logra analizar a detalle una de las principales características que distingue los procesos formativos de las y los estudiantes de las universidades interculturales: su temprana y prolongada inmersión en procesos de investigación extraáulica y de vinculación comunitaria, en la que se conjugan objetivos de profesionalización con aspiraciones de compromiso social con los actores locales.

En segundo lugar, Carlos Luis Maldonado Ramírez aporta en su artículo "La investigación como método pedagógico: hacia la innovación docente en contextos comunales. El caso de la Unidad de Estudios Superiores de Alotepec Mixe, Oaxaca" un análisis de las diferentes experiencias formativas por las que transitan las y los estudiantes de la UESA en el ámbito familiar y comunitario, en el ámbito de los Bachilleratos Integrales Comunitarios que cursaron previamente y, finalmente, en el ámbito de la propia licenciatura en educación media superior comunitaria. La resultante triangulación, vislumbra tensiones y contradicciones entre estos ámbitos, pero a la vez ilustra el novedoso perfil de educador/a comunitario/a que egresa de esta universidad.

La tercera experiencia proviene de la sede Totonacapan de la UVI, para la cual, Sara Itzel Arcos Barreiro, en su artículo "Organización social y participación: diseño innovador de la experiencia educativa que fortalece el diálogo de saberes en la Universidad Veracruzana Intercultural”, reconstruye detalladamente el proceso de diseño, implementación y evaluación de una materia-clave para la formación de las y los futuros licenciados en gestión intercultural para el desarrollo que ofrece la UVI. La articulación curricular de saberes académicos y comunitarios, el abordaje inter o transdisciplinario de estos saberes, así como la estrecha relación que se logra generar entre docencia, investigación y vinculación, demuestran cuánto se distingue este tipo de "materia" de las asignaturas que clásicamente imparten las universidades en carreras más convencionales.

Para el caso de la Universidad Intercultural Indígena de Michoacán, Carlos Montalvo Martínez ofrece en su trabajo "La educación intercultural como educación para la conciliación del antagonismo de clases y el rol social del PA en Gestión Comunitaria y Gobiernos Locales de la UIIM" un recorrido histórico de la educación intercultural y sus antecesores en ese estado, en el contexto de conflictividad socioeconómica y de exclusión política que predomina en las regiones indígenas, para luego desembocar en un análisis de la pertinencia de la licenciatura en Gestión Comunitaria y Gobiernos Locales que la UIIM imparte. A partir de su valoración crítica de programas diseñados desde una visión externa, y a menudo armonizante de la interculturalidad, el autor genera una propuesta de transitar hacia una intercomunalidad concebida desde dentro que asegure la participación plena de las autoridades comunales en la gestión de la propia universidad intercultural.

La aportación que la investigación artística y escénica pueda hacer a la innovación docente, constituye el núcleo de los dos siguientes 
artículos. En "Corporalidad, creación escénica y teatro comunitario en la Universidad Veracruzana Intercultural (UVI)”, Claudia P. Eguiarte Espejo nos presenta el proceso de consolidación del teatro comunitario, a partir de un grupo bilingüe -náhuatl-castellano- de estudiantes, docentes y egresadas/os de la sede Grandes Montañas de la UVI, que logran animar, promover y revitalizar el uso del náhuatl, a la vez que contribuyen a incrementar la concientización y la participación local en la resolución de problemas con pertinencia cultural. La metodología de investigacióncreación participativa y la recuperación del cuerpo y del espacio de lo propio, constituyen herramientas sumamente novedosas para conjugar la docencia, la investigación y la vinculación desde un enfoque bilingüe e intercultural.

Por su parte, Carlos Alberto Cruz González muestra en su trabajo "Expresiones del Arte Totonaco: una experiencia de gestión del aprendizaje" cómo aprovechar una materia curricular para generar una intervención educativa que cuestiona y subvierte la estructura vertical de los saberes universitarios al abrirlos, en este caso, a los saberes artísticos que aportan las y los ancianos miembros del Centro de Artes Indígenas de El Tajín. Los aprendizajes extra-áulicos desafían los saberes-haceres tanto de estudiantes de la Sede Totonacapan de la UVI como de estudiantes de carreras convencionales de la Universidad Veracruzana, que participan conjuntamente en esta experiencia transdisciplinaria e intercultural.

Pasando de las universidades interculturales a las escuelas formadoras de docentes con enfoque bilingüe e intercultural, en su trabajo "Geografía y territorialidad indígena: Innovación docente en la Escuela Normal Bilingüe e Intercultural de Oaxaca" Benjamín Maldonado Alvarado estudia monográficamente cómo la docente de la materia de geografía logra transformar el saber académico de esta disciplina, en una serie de saberes contextualizados, territorializados desde la propia realidad cultural y lingüística de cada estudiante. Recurriendo a mapas conceptuales, entrevistas con ancianas y ancianos, la confección de calendarios comunitarios, entre otros métodos didácticos, la profesora resignifica la materia convirtiéndola en una serie de "geografías sagradas" producto de los saberes propios de las y los estudiantes.

Frente a este tipo de proyecto exitoso de innovación, por último, María Isabel Ramírez Duque analiza en su contribución "El carácter colonial del poder y del saber en el devenir de la Especialidad de Educación Intercultural Bilingüe de la Universidad Pedagógica Experimental Libertador de Venezuela" el origen de la educación intercultural y bilingüe en el contexto venezolano, para luego evaluar críticamente porqué, en el caso de la mencionada especialidad, aún no se logra poner en diálogo ontologías y epistemologías diversas. Un excesivo énfasis en lo discursivo y una falta de apropiación práctica y curricular de los conocimientos indígenas, reflejan, según la autora, los pendientes de descolonización a los que se enfrentan las instituciones formadoras de profesionales de la educación intercultural y bilingüe -un desafío igualmente vigente en el caso de las IES mexicanas. 
Una característica particular que nos parece muy destacable en el conjunto de artículos aquí presentados, consiste en el hecho de que todos los autores y autoras provienen de la IES estudiada o vienen desde hace años colaborando muy estrechamente con la misma. La gran mayoría de los trabajos procede de investigaciones empíricas, áulicas y extra-áulicas, que han realizado los propios autores y autoras como docentes-investigadores/as de sus prácticas académicas. Ubicándose por tanto, metodológicamente, entre la investigación cualitativa y etnográfica, por un lado, y la investigación-acción participativa por el otro, los resultados de investigación aquí publicados tienen una novedosa y creativa dimensión autobiográfica, autoetnográfica o, por lo menos, autorreflexiva. Consideramos que estas aportaciones metodológicas a la investigación sobre la docencia con enfoque intercultural, no solamente complementan la innovación docente aquí analizada, sino que abren pistas para futuras innovaciones metodológicas para la investigación que estamos realizando y/o pretendemos realizar con un enfoque intercultural, dialógico y descolonial.

\section{Referencias}

Boaventura de Sousa, S. (2005). La Universidad en el siglo XXI:para una reforma democrática y emancipadora de la Universidad. México: UNAM- CIICH.

Carbonell, J. (2015). Pedagogías del siglo XXI: alternativas para la innovación educativa. Barcelona: Octaedro

Casillas, L. y Santini, L. (2006). Universidad Intercultural: modelo educativo. México: SEP-CGEIB

Clark, R. (2000). Creando universidades innovadoras: estrategias organizacionales para la transformación. México: UNAM - Porrúa

Cuerpo Académico Estudios Interculturales (2016a). Universidades Interculturales en México: balance de una década. I Seminario de investigación. Universidad Veracruzana, Xalapa, Veracruz, México. Recuperado de: https://www.uv.mx/iie/files/2013/02/I-seminario-Univ ersidades-Interculturales-Documento-de-trabajo.pdf

Cuerpo Académico Estudios Interculturales (2016b). UNIVERSIDADES INTERCULTURALES EN MÉXICO: BALANCE DE UNA DÉCADA. II Seminario de investigación. Universidad Veracruzana, Xalapa, Veracruz, México. Recuperado de: https://www.uv.mx/iie/files/2013/02/II-semin ario-Universidades-Interculturales-Documento-de-trabajo.pdf

Dietz, G. (2014). Universidades Interculturales en México. CPUe. Revista de Investigación Educativa 19. Recuperado de: http://revistas.uv.mx/index.p $\mathrm{hp} / \mathrm{cpue} /$ article/view/974

Gazzola, A. L. y Didriksson, A. (Eds.), (2008). Tendencias de la educación superior en América Latina y el Caribe. Caracas: UNESCO-IESALC.

Ibarra, E. (2002). La "nueva universidad" en México: transformaciones recientes y perspectivas. Revista Mexicana de Investigación Educativa, 7 (14), 75-105.

Inayatullah, S. y Gidley, J. (2003). La universidad en transformación: perspectivas globales sobre los futuros de la universidad. Barcelona: Pomares. 
Mateos, L. S. y Dietz, G. (2013). Universidades Interculturales en México. En: M. Bertely, G. Dietz y G. Díaz (Coords.), Multiculturalismo y educación 2002-2011, (pp. 349-381). México: COMIE Y ANUIES.

Mateos, L. S. y Dietz, G. (Coords.), (2016). Universidades interculturales en México: balance crítico de la primera década. Revista Mexicana de Investigación Educativa, 21 (70), México: COMIE.

Mateos, L. S., Mendoza G. y Dietz, G. (2013). Diversidad e interculturalidad en la educación superior convencional. En: M. Bertely, G. Dietz y G. Díaz (Coords.), Multiculturalismo y educación 2002-2011, (pp. 307-347). México: COMIE Y ANUIES.

Mato, D. (Ed.), (2009). Instituciones Interculturales de Educación Superior en América Latina: procesos de construcción, logros, innovaciones y desafios. Caracas: UNESCO-IESALC.

Rojas, A. y González, E. (2016). El carácter interactoral en la educación superior con enfoque intercultural en México. Limina R, 14 (19), 73-91.

Schmelkes, S. (2008). Creación y desarrollo inicial de las universidades interculturales en México: problemas, oportunidades, retos. En: D. Mato (Coord.), Diversidad Cultural e Interculturalidad en Educación Superior: experiencias en América Latina (pp. 329-337). Caracas: UNESCO-IESALC.

Shore, Ch. y Wright, S. (2015). Governing by numbers: audit culture, rankings and the new world order. Social Anthropology / Anthropologie Sociale, 23 (1), 22-28.

\section{Notas}

1 Cfr. por ejemplo las visiones panorámicas aportadas por Schmelkes (2008), Mateos y Dietz (2013, 2016), Dietz (2014) y Rojas y González (2016). 